\title{
A RBSDES APPROACH FOR THE NUMERICAL EVALUATION OF THE AMERICAN OPTION PRICING PROBLEM
}

\author{
Roberta Di Franco \\ Department of Computer Sciences \\ University of Verona \\ Strada le Grazie 15, 37134, Verona, ITALY
}

\begin{abstract}
The problem of pricing American type options is a typical example of a non linear problem characterized by the absence of closed expressions for its evaluation. Therefore, during recent years, such an issue has been approached, both deterministically and randomly, from the algorithmic point of view, trying to derive suitable numerical approximations. In this paper, starting from the aforementioned solutions, we review some computational, stochastic inspired, methods, mainly based on the the existing link between the above recalled pricing task, and the theory of Reflected Backward Stochastic Differential Equations (BSDEs). In particular we show how suitable numerical schemes can be developed within the SBDEs framework by mean of quantization techniques as well as considering Monte Carlo methods.
\end{abstract}

AMS Subject Classification: 60H15, 60H35, 90-08, 91G20, 91G80, 91B25

Key Words: American options, reflected stochastic backward differential equations, stochastic quantization, Monte Carlo methods

\section{Introduction}

There is a vast literature about Backward Stochastic Differential Equations (BSDEs) and their applications. The fundamental definitions and first results concerning BSDES have been introduced by Bismut, see [6], for the linear case and by Pardoux and Peng, see [31], in the general case. Duffie and Epstein $[17,18]$, have shown that the BSDEs point of view gives a simple formulation

Received: July 27, 2016

Published: September 10, 2016 (c) 2016 Academic Publications, Ltd.

url: www.acadpubl.eu 
of recursive utilities and their properties. In fact, recursive utility corresponds to the solution of a particular BSDE associated with a generator which does not depend on z-component, see below for more details. Moreover, the BSDEs theory has been extensively used in stochastic control, see, e.g., [3] and references therein, as they appear as adjoint equations in the stochastic version of Pontryagin maximum principle, and in Mathematical Finance, since any pricing problem by replication can be written in terms of linear BSDEs, or non-linear BSDEs when portfolios constraints are taken into account, see $[19,11,12,13,14]$.

More recently, El Karoui, Kapoudjian, Pardoux, Peng and Quenez generalized these results to BSDEs with reflection, see [20], that is, to a setting with an additional continuous, increasing process added in the equation; the function of this additional process is to keep the solution above a certain prescribed lowerboundary process and to do so in a minimal fashion. Moreover, these authors make the crucial observation that the solution is the value function of an optimal stopping problem. Those RBSDEs have been intensively investigated due to their connections with financial mathematics, optimal control and stochastic game. In [1] showed a new construction of the solution of RBSDEs using a maximum principle. This appears as an alternative to the classical penalization method used in [19]. Penalization method produces the solution of a RBSDE as an increasing limit of a sequence of solution of standard (non-reflected) BSDEs. In [23] the author gives an application linked to the stochastic mixed control problems, while in [21] it has been solved the problem of existence and uniqueness of solution to RBSDEs with jumps. Note that the latter problem can be also studied by the point of view of semilinear stochastic evolution equations, also in connection with approaximating procedures, as it is done, e.g., in [27]

Concerning the connection with the Mathematical Finance framework, we recall what follows: the holder of a stock option has the right either sell or buy a certain quantity of a company's stock by a certain date at a given price. The price in the contract is known as the strike price and the date is known as the expiration date, or maturity.

A put, resp. a call, option gives the holder the right to sell, resp. to buy, the underlying asset. There exists several types of stock option, spanning from plain Vanilla, or European, ones, to highly structured derivatives. Between latter pletora of possibilities, one of the most traded type is represented by American option. Unlike the European stock option, which can be exercised at maturity only, American options can be exercised at any time up to maturity, which implies that the problem of American option valuation does not have a closed-form solution which, instead, can be given exploiting numerical meth- 
ods. In one-dimension, the most popular approach to American option pricing and hedging is represented by the implementation of the dynamic programming formula on a binomial tree, originally initiated by Cox-Ross \& Rubinstein as an elementary alternative to continuous time Black \& Scholes model. These methods are quite efficient to handle vanilla American options on a single asset but they quickly become intractable as the number of underlying assets increases. In order to increase the efficiency and speed of the previous method, different proposal have been studied, leading to the specification of three main family of computational approaches: Binomial Trees, Finite Difference methods and Monte Carlo methods. Note also that previous approaches can be partially applied to the study of financial time series in order to better understand the comovements of assets dynamics and related (stochastic) volatilities of the markets they are traded in. In particular, latter type of problem can be effectively analysed by mean of tecniques as the Gibbs sampling, or the regime switching one, see, e.g., [15, 16], and references therein.

Concerning the Monte Carlo methods, one can use them when aiming to solve a problem by simulating the underlying process, e.g., a stock, and then calculate the average of some function of the simulated process. A great advantage of the Monte Carlo approach is its flexibility, since it can treat a large set of fundamental problems in statistics, biology, numerical analysis, etc. Moreover, Monte Carlo methods can also handle high dimensional problems effectively. The ideas behind the Monte Carlo method was developed in the 40's by Stanislaw Ulam with together with the help of John von Neumann. Their ideas were later used within the Manhattan project, namely the research program that lead to the development of the first atomic bombs during the second World War.

In the 60's the first financial applications of Monte Carlo methods was suggested by Herts, see [24], and Boyle, see [10]. Then, Bossaerts [9] and Tilley [33], suggested that Monte Carlo methods could be also used to price American style derivatives. Nevertheless, such type of numerical approaches are outperformed by ad hoc solutions at least in some particular frameworks as those when once for all computations can be realized. The latter is the case, just to give concrete examples, of the evaluation of expectation of known random variables by mean of polynomial chaos decomposition, as happens for relevant financial models like, e.g., the Vasicek model, or the Cox-IngersollRoss (CIR) model, see [8]. Concerning the quantization method, see, e.g., [7] and references therein, it is worth to mention that it is well-adapted for the pricing and hedging of American options. Its purpose is to compute a large number of conditional expectations by projection of the diffusion on optimal 
grid designed to minimize the (square mean) projection error.

Quantization methods have been also extensively investigated in Information Theory and signal processing [22], while, since the early 2000's, the associated infinite dimensional setting has been investigated from both theoretical and numerical point of view, with a special attention paid to functional quantization, see $[26,29]$. Other types of quantization method, as Marginal quantization and Markovian quantization, were proposed in [28], the first one, relies on an optimal approximation of the marginal distributions of the process, while the second one, looks for an optimal approximation of transition probabilities of the Markov process at some points. And more recently, quantization was introduced in numerical probability to devise numerical integration method [30] and to solve multidimensional stochastic control problems such as swing options, see [4].

In the present paper we shall exploit the approach mainly developed in $[2,34]$, to the links between the RBSDEs theory, Monte Carlo and quantization method, to price American options.

In particular, the paper is organized as follows: in Section 2 we give some basic notions on BSDEs, as, e.g., existence and uniqueness of solutions and the comparison principle; in Section 3 we report almost the same basic notions, but in the RBSDEs setting, in Section 4 we will show some possible applications of the theoretical techniques developed for BSDEs and RBSDEs in Finance, in particular in Subsection 4.1, resp. in Subsection 4.2, we present the link between RBSDEs and American option, resp. the link between quantization method and RBSDE, while the application of the Monte Carlo approach to the same problem will be treated in Subsection 4.3.

\section{Notations}

Throughout the work we will make use of the following notations

- $C_{b}^{\infty}\left(\mathbb{R}^{d}\right)$ denotes the set of functions infinitely differentiable with bounded differentials (so that they have at most linear growth).

- The letters $\mathrm{C}$ and $\mathrm{K}$ denote positive real constants that may vary from line to line.

- | . | will denote the Euclidean norm and "." the inner product on $\mathbb{R}^{d}=$ $\mathbb{R}^{1 \otimes d} .\|M\|:=\sup _{|x| \leq 1}|M x|$ will denote the operator norm of the matrix $M \in \mathbb{R}^{d \otimes q}$ (d rows, q columns) and $M^{*}$ its transpose (sometimes also denoted by $M^{T}$ ). In particular $x \cdot y=x^{*} y\left(\right.$ or $\left.x . y=x^{T} y\right)$. 


\section{Backward Stochastic Differential Equations}

Roughly speaking, BSDEs are used to analyse what happens if instead of imposing an initial condition for a Stochastic Differential Equation (SDE), one considers a terminal condition.

Let $\mathrm{W}$ be a 1-dimensional Brownian motion defined on a probability space $(\Omega, F, \mathbb{P})$, and let us consider the filtration:

$$
F_{t}^{W}:=\sigma\left(W_{s} \mid s \in[0, t]\right), F_{t}:=F_{t}^{W} \vee N_{p}, t \geq 0
$$

We fix a finite horizon $0<T<+\infty$, and $f, g: \mathbb{R} \rightarrow \mathbb{R}$ to be Lipschitz functions. The SDE:

$$
d Y_{t}=f\left(Y_{t}\right) d t+g\left(Y_{t}\right) d W, t \in[0, T],
$$

has a unique solution $Y \in S^{2}([0, T])$, provided we set an initial condition $Y_{0}=x$. Recall that $Y_{T} \in L^{2}\left(\Omega, F_{T}, \mathbb{P}\right)$. We use the martingale representation theorem $(\mathrm{MRT})$, since $\xi \in L^{2}\left(\Omega, F_{T}, \mathbb{P}\right)$ and $\left(Y_{t}\right)_{t \geq 0}=\left\{\mathbb{E}\left[\xi \mid F_{t}\right]\right\}_{t \geq 0}$ is a squareintegrable martingale; by MRT, there exists a process $Z \in L_{a d}^{2}([0, T] \times \Omega)$ such that:

$$
Y_{t}=\mathbb{E}[\xi]+\int_{0}^{t} Z_{s} d W_{s}, t \in[0, T]
$$

namely

$$
\left\{\begin{array}{l}
d Y_{t}=Z_{t} d W_{t}, t \in[0, T] \\
Y_{T}=\xi
\end{array} .\right.
$$

In general, instead of (1), we consider equations of the following form:

$$
\left\{\begin{array}{l}
d Y_{t}=f\left(Y_{t}\right) d t+g\left(Y_{t}\right) d W_{t}+Z_{t} d W_{t}, t \in[0, T] \\
Y_{T}=\xi \in L^{2}\left(\Omega, F_{T}, \mathbb{P}\right)
\end{array},\right.
$$

where the pair $(Y, Z) \in S^{2}([0, T]) \times L_{a d}^{2}([0, T] \times \Omega)$ is the unknown. Substituting $\tilde{Z}_{t}:=g\left(Y_{t}\right)+Z_{t}$, we see that it is enough to look for the solutions of the following equation:

$$
\left\{\begin{array}{l}
d Y_{t}=f\left(Y_{t}\right) d t+\tilde{Z}_{t} d W_{t}, t \in[0, T] \\
Y_{T}=\xi \in L^{2}\left(\Omega, F_{T}, \mathbb{P}\right)
\end{array},\right.
$$

defined to be the BSDE we are interested in.

Let us consider the following general form for a BSDE

$$
\left\{\begin{array}{l}
d Y_{t}=-f\left(t, Y_{t}, Z_{t}\right) d t+Z_{t} d W_{t}, t \in[0, T] \\
Y_{t}=\xi \in L^{p}\left(\Omega, F_{T}, \mathbb{P}\right)
\end{array},\right.
$$


under suitable assumptions on the driver $\mathrm{f}$ and final data $\xi$, which were described below, and for $p>2$.

In what follows we report a general result about existence and uniqueness of the solution of a BSDE when the coefficient are Lipschitz-continuous. Let $\left(\Omega, F_{T}, \mathbb{P}\right)$ be a complete probability space, W a d-dimensional Brownian motion defined on $\left(\Omega, F_{T}, \mathbb{P}\right), F_{t}=F_{t}^{W} \vee N_{p}, 0 \leq t \leq T, \mathbb{F}=\left(F_{t}\right)_{t \in[0, T]}$. We impose the following conditions on the coefficients:

(C1): The driver $f: \Omega \times[0, T] \times \mathbb{R}^{k} \times \mathbb{R}^{k \times d} \rightarrow \mathbb{R}^{k}$ is measurable and:

1. $f(\cdot, \cdot, y, z)$ is progressively measurable for every $(y, z) \in \mathbb{R}^{k} \times \mathbb{R}^{k \times d}$;

2. $\mathbb{E}\left[\int_{0}^{T}|f(t, 0,0)|^{2} d t\right]<+\infty$;

3. $\exists L>0:\left|f(\omega, t, y, z)-f\left(\omega, t, y^{\prime}, z^{\prime}\right)\right| \leq L\left(\left|y-y^{\prime}\right|+\left|z-z^{\prime}\right|\right)$ $\forall y, y^{\prime} \in \mathbb{R}^{k}, \forall z, z^{\prime} \in \mathbb{R}^{k \times d}, P(d \omega) d t-$ a.e. on $\Omega \times[0, T] ;$

(C2): $\xi \in L^{2}\left(\Omega, F_{T}, \mathbb{P}, \mathbb{R}^{k}\right)$.

Theorem. Under the conditions $C 1$ and $C 2$, the equation (2) has unique solution $(Y, Z) \in B^{2}=L_{a d}^{2}\left(\Omega \times[0, T], \mathbb{R}^{k}\right) \times L_{a d}^{2}\left(\Omega \times[0, T], \mathbb{R}^{k \times d}\right)$.

Proof. see Section 3 in [31]. Or for a shorter direct proof using useful a priori estimates, see Theorem 2.1 in [19].

A second, particularly relevant, result is the so called Comparison Principle, which is of great interest when one has to compare the solutions of two BSDEs.

Comparison Principle. Let $f^{i}: \Omega \times[0, T] \times \mathbb{R} \times \mathbb{R}^{d} \rightarrow \mathbb{R}, i=1,2$ two functions satisfying $(C 1)$ and $\xi^{1}, \xi^{2} \in L^{2}\left(\Omega, F_{T}, \mathbb{P}\right)$. For $i=1,2$, let $\left(Y^{i}, Z^{i}\right) \in B^{2}$ be the unique solution of $\operatorname{BSDE}\left(f^{i}, \xi^{i}\right)$. If:

- it holds $f^{1}\left(t, Y_{t}^{1}, Z_{t}^{1}\right) \leq f^{2}\left(t, Y_{t}^{1}, Z_{t}^{1}\right)$, dtdP-a.e. on $[0, T] \times \Omega$ or $f^{1}\left(t, Y_{t}^{2}, Z_{t}^{2}\right) \leq f^{2}\left(t, Y_{t}^{2}, Z_{t}^{2}\right)$, dtdP-a.e. on $[0, T] \times \Omega$;

- $\xi^{1} \leq \xi^{2}$

then $Y_{t}^{1} \leq Y_{t}^{2} \forall t \in[0, T], P$-a.s.

Proof. see Section 2.2 in [19]. 


\section{Reflected Backward Stochastic Differential Equations}

The notion of Reflected Backward Stochastic Differential Equation (RBSDE) has been introduced by El Karoui in [20], defining BSDEs characterized by an increasing process $K$ introduced in order to force the solution $Y$ of the (original) BSDE to remain larger than a given process $S$ representing an obstacle or barrier.

In order to provide the basics notions and results for RBSDEs, let us start by fixing a d-dimensional standard Brownian motion, $W_{t}, 0 \leq t \leq T$, defined on a complete probability space $\left(\Omega, F_{T}, \mathbb{P}\right)$. Moreover, let $F_{t}, 0 \leq t \leq T$ be the natural filtration of $W_{t}$ and let $P$ be the $\sigma$-algebra of predictable subsets of $\Omega \times[0, T]$.

Further, consider teh following notations:

$\mathbb{L}^{2}=\left\{\xi\right.$ an $F_{t}$-measurable random variable such that $\left.\mathbb{E}\left[|\xi|^{2}\right]<+\infty\right\} ;$

$\mathbb{H}^{2}=\left\{\phi_{t}, 0 \leq t \leq T\right.$ a predictable process such that $\mathbb{E}\left[\int_{0}^{T}\left|\phi_{t}\right|^{2} d t\right]<$ $+\infty\}$;

$\varrho^{2}=\left\{\phi_{t}, 0 \leq t \leq T\right.$ a predictable process such that $\mathbb{E}\left[\sup _{0 \leq t \leq T}\left|\phi_{t}\right|^{2}\right]<$ $+\infty\}$.

Previous definitions can be re-read in terms of the following three objects:

1. the first is a terminal value $\xi$ such that $\xi \in \mathbb{L}^{2}$;

2. the second is a coefficient $\mathrm{f}$, which is a map: $f:[0, T] \times \mathbb{R} \times \mathbb{R}^{d} \rightarrow \mathbb{R}$, such that:

(a) $\forall(y, z) \in \mathbb{R} \times \mathbb{R}^{d}, f(\cdot, y, z) \in \mathbb{H}^{2}$,

(b) for some $K_{i} 0$ and all $y, y^{\prime} \in \mathbb{R}, z, z^{\prime} \in \mathbb{R}^{d}$, a.s.

$\left|f(t, y, z)-f\left(t, y^{\prime}, z^{\prime}\right)\right| \leq K\left(\left|y-y^{\prime}\right|+\left|z-z^{\prime}\right|\right)$,

3. the third is an obstacle or barrier, $S_{t}, 0 \leq t \leq T$, which is a continuous progressively measurable real-valued process satisfying $\mathbb{E}\left[\sup _{0 \leq t \leq T}\left(S_{t}^{+}\right)^{2}\right]<$ $+\infty$.

Then, a solution of a given RBSDE is a triple of process $(Y, Z, K)$, taking values in $\mathbb{R} \times \mathbb{R}^{d} \times \mathbb{R}_{+}$, and satisfying:

$$
Y_{t}=\xi+\int_{t}^{T} f\left(s, Y_{s}, Z_{s}\right) d s+K_{T}-K_{t}-\int_{t}^{T} Z_{s} d W_{s}, \forall t \in[0, T] .
$$

The additional process $K$ is continuous and non-decreasing, moreover its role is to push upwards the process $Y$ in order to keep it above the barrier $\mathrm{S}$, provided that: 
i) $(Y, Z) \in B^{2}=L_{a d}^{2}([0, T] \times \Omega) \times L_{a d}^{2}\left([0, T] \times \Omega\right.$; $\left.\mathbb{R}^{d}\right)$ and $K \in S^{2}([0, T])$ is a non-decreasing process with $K_{0}=0$;

ii) $Y_{t} \geq S_{t} \forall t \in[0, T]$ and $\int_{0}^{T}\left(Y_{t}-S_{t}\right) d K_{t}=0$.

The latter implies that the process $K$ acts only when $Y$ reaches the barrier $S$, but in a minimal way.

Theorem. Under the above conditions, if $\xi \geq S_{T}$, then the RBSDE has a unique solution.

Proof. See Section 2 in [20].

To what concerns the RBSDE counterpart of the BSDE-comparison principle, we have :

Comparison Principle for RBSDEs. Let $(\xi, f, S)$ and $\left(\xi^{\prime}, f^{\prime}, S^{\prime}\right)$ be two sets of data taken according to the object specifications given at points [1], [2], [3], above, but with the exception that the Lipschitz condition [2] could be satisfied by either $f$ or $f^{\prime}$ only. Suppose in addition that the following inequalities hold

- $\xi \leq \xi^{\prime}$ a.s.

- $f(t, y, z) \leq f^{\prime}(t, y, z) d P \times d t$ a.e. $\forall(y, z) \in \mathbb{R} \times \mathbb{R}^{d}$

- $S_{t} \leq S_{t}^{\prime}$ a.s. $0 \leq t \leq T$.

Let $(Y, Z, K)$ be a solution of the RBSDE with data $(\xi, f, S)$ and $\left(Y^{\prime}, Z^{\prime}, K^{\prime}\right)$ a solution of the RBSDE with data $\left(\xi^{\prime}, f^{\prime}, S^{\prime}\right)$. Then:

$$
Y_{t} \leq Y_{t}^{\prime}, 0 \leq t \leq T \text { a.s. }
$$

Proof. see Theorem 4.1 in [20].

\section{Application in Finance}

\subsection{RBSDEs and American Option}

We start this section giving a short introduction to the Snell envelope technique, which plays an important role in evaluting American type options, indeed the valuation of American options is a special case of the Snell envelope, see [32]. 
Definition. If $X=\left(X_{n}\right)_{n=0}^{N}$ is a sequence of random variables, adapted to a filtration $F_{n}$ with $\mathbb{E}\left[\left|X_{n}\right|\right]<\infty$, the sequence $Z=\left(Z_{n}\right)_{n=0}^{N}$ defined by:

$$
\left\{\begin{array}{l}
Z_{N}=X_{N} \\
Z_{n}=\max \left(X_{n}, \mathbb{E}\left[Z_{n+1} \mid F_{n}\right]\right), n \leq N-1,
\end{array},\right.
$$

is called the Snell envelope of X.

The sequence $\mathrm{Z}$ can be considered to be an optimal value process:

$$
Z_{n}=e s s \sup _{n \leq \tau \leq T} \mathbb{E}\left[X_{\tau} \mid F_{n}\right]
$$

The recursion (3) defines the optimal stopping strategy to find the optimal value $Z_{n}$, and it is usual to refer to it, as the method of dynamic programming. The time that maximizes the essential supreum (4), is called an optimal stopping time and is denoted $\hat{\tau}_{n}$. We also recall that:

Definition. Let $(\Omega, F, \mathbb{P})$ be a probability space and $N_{t}$ be an increasing family of $\sigma$-algebras of subset of $\Omega$. A function $\tau: \Omega \rightarrow[0, \infty]$ is called a stopping time with respect to $N_{t}$ if:

$$
\{\omega \mid \tau(\omega) \leq t\} \in N_{t}, \forall t \geq 0 .
$$

Concerning the financial framework, let us recall that the holder of an American option has the option to either buy (call) or sell (put) the underlying asset $S$ at a certain, fixed a priori, strike price $K$, at an optional time $\tau \leq T, T$ being the terminal, or maturity, time of the investment. The related payoff function is given by:

$$
\Phi(S)=\left\{\begin{array}{ll}
\left(S_{\tau}-K\right)^{+} & \text {for call } \\
\left(K-S_{\tau}\right)^{+} & \text {for put }
\end{array} .\right.
$$

Latter definition implies that the value of an American option is the value achieved by exercising optimally. Finding the optimal exercise rule is an optimal stopping problem. Indeed, for each value of $S_{t}, t=T, \ldots, 0$, we have to determine the price of the option and check if it is favorable to exercise. This problem is known as a free boundary problem. At each time $t$ we have two possibilities: hold on to the option or exercise it. The price, which we can denote $S_{\tau}$, is called the optimal exercise price. We do not know $S_{\tau}$ a priori and thus we do not know how to apply the boundary conditions, why this problem is referred 
to as a free boundary problem. Hence, the problem of pricing an American option is to find the optimal expected discounted payoff:

$$
\sup _{\tau \in\urcorner} e^{r(T \tau)} \mathbb{E}\left[\Phi\left(S_{\tau}\right)\right]
$$

where 7 is the class of admissible stopping times, taking values in $[0, T]$.

So we restate that, finding a fair value is not an easy task due to the complexity of the derivative, that is, in the case of simple derivatives, we can derive the value in a closed-form solution, for the American option we requires numerical procedures.

Therefore, in what follows, we state the model linking the American options pricing task to the theory of RBSDEs.

In order to achieve such a goal, let us start considering a stock market model constituted by a non-risky asset (bond) $S^{0}$, and a finite family of risky assets $S^{i} \mathrm{i}=1, \ldots, \mathrm{m}$. The price of one bond unit is given by:

$$
d S_{t}^{0}=S_{t}^{0} r_{t} d t, t \geq 0,
$$

where $r$ is the (risk free) interest rate, while the price processes of the risky assets are modelled by:

$$
d S_{t}^{i}=S_{t}^{i}\left(\mu_{t}^{i} d t+\sigma_{t}^{i} d W_{t}\right), t \geq 0,
$$

where $\mathrm{W}$ is a d-dimensional Brownian motion. We assume that $S_{0}^{0}>0$ and $S_{0}^{i}>0, \mathrm{i}=1, \ldots, \mathrm{m}$. The processes $r$ and $\mu^{i}$ are supposed progressively measurable and bounded. Here, $\sigma^{i}$ are known as the volatilities of the prices, and we suppose that the matrix $\sigma=\left(\sigma^{i}\right)_{1 \leq i \leq m}$ is invertible, hence, in particular $m=d$., with its inverse bounded, which corresponds to the fact that the market is arbitrage-free and complete.

Let $\bar{\pi}=\left(\pi_{t}^{0}, \pi_{t}^{1}, \ldots, \pi_{t}^{m}\right)_{0 \leq t \leq T}$ be a portfolio, where the processes $\pi^{0}$ and $\pi^{i}$, $\mathrm{i}=1, \ldots, \mathrm{m}$; are the amounts invested in the bond and the risky asset $i$, respectively. The value of the portfolio $\bar{\pi}$ is given by the process:

$$
V_{t}=V_{y}^{\bar{\pi}}:=\pi_{t}^{0}+\pi_{t}^{1}+\ldots+\pi_{t}^{m}, t \in[0, T] .
$$

We recall that a portfolio is called admissible if $\bar{\pi}$ is progressively measurable and if:

$$
\begin{aligned}
& \int_{0}^{T}\left|\pi_{t}^{0}\right| d t<+\infty, \int_{0}^{T}\left|\pi_{t}^{i} \sigma_{t}^{i}\right|^{2} d t<+\infty \\
& \forall i=1, \ldots, m P-\text { a.s. }, V_{t}^{\bar{\pi}} \geq 0, \forall t \in[0, T] .
\end{aligned}
$$


An admissible portfolio $\bar{\pi}$ is called self-financing if, taking $\sigma^{0}=0$, its value $V_{t}^{\bar{\pi}}$ satisfies:

$$
\begin{aligned}
d V_{t}= & \pi_{t}^{0} \frac{d S_{t}^{0}}{S_{t}^{0}}+\pi_{t}^{1} \frac{d S_{t}^{1}}{S_{t}^{1}}+\ldots+\pi_{t}^{m} \frac{d S_{t}^{m}}{S_{t}^{m}}=r_{t} V_{t} d t+\sum_{i=1}^{m}\left(\pi_{t}^{i}\left(\mu_{t}^{i}-r_{t}\right) d t+\pi_{t}^{i} \sigma_{t}^{i} d W_{t}\right)= \\
& =r_{t} V_{t} d t+\pi_{t}\left(\mu_{t}-r_{t}\right) d t+\pi_{t} \sigma_{t} d W_{t}=\left(r_{t} V_{t}+\pi_{t} \sigma_{t} \theta_{t}\right) d t+\pi_{t} \sigma_{t} d W_{t},
\end{aligned}
$$

where $\pi_{t}=\left(\pi_{t}^{1}, \ldots, \pi_{t}^{m}\right)$ is the risky portfolio, while we indicate by $\theta_{t}=(\sigma)^{-1}\left(\mu_{t}-\right.$ $\left.r_{t}\right)$ is the risk premium vector. The set of admissible self-financing portfolios will be denoted by $A$.

It is customary to write $(V, \pi) \in A$, meaning $\left(\pi^{0}, \pi\right) \in A$ and $\pi_{t}^{0}=V_{t}-$ $\sum_{i=1}^{m} \pi_{t}^{i}, t \in[0, T]$.

For our purpose, the wealth-portfolio $(V, \pi)$ has to satisfy:

$$
-d V_{t}=b\left(t, V_{t}, \pi_{t}\right) d t-\pi_{t} \sigma_{t} d W_{t}, t \geq 0,
$$

where $b$ is convex and Lipschitz, with respect to $(x, \pi)$. Indeed, in the standard case, we have:

$$
b(t, x, \pi)=-r_{t} x-\pi \sigma_{t} \theta_{t} .
$$

When the market is constrained, namely when the interest rates are not the same whether we borrow or invest money, then the function $b$ takes the alternative form:

$$
b(t, x, \pi)=-r_{t} x-\pi \sigma_{t} \theta_{t}+\left(R_{t}-r_{t}\right)(x-<\pi, 1>)^{-},
$$

where $R_{t}$ and $r_{t}$ are the spot rates to borrow, respectively to invest. Additionally, we assume that:

$$
\mathbb{E}\left[\int_{0}^{T}|b(t, 0,0)|^{2} d t\right]<+\infty,
$$

where $\left(\sigma_{t}\right)_{0 \leq t \leq T}$, which represents the volatility matrix of the $n$ risky assets, is invertible and its inverse $\left(\sigma_{t}\right)^{-1}$ is bounded. Without loss of generality, we can even suppose that $\sigma_{t}=I$.

It follows that we are concerned with the problem of pricing an American option at time $t=0$. When it is exercised at a stopping time $\tau \leq T$, the yield is given by:

$$
\tilde{P}_{\tau}:=P_{\tau} 1_{(\tau<T)}+\xi 1_{(\tau=T)},
$$

Recall that, in case of American put options, we have $P_{t}=\left(K-S_{T}^{1}\right)^{+}, \xi P_{T}$, so $\tilde{P}_{\tau}=P_{\tau}$. 
Then there exists a unique pair $\left(V_{p}\left(\tau, \tilde{P}_{\tau}\right), \pi_{p}\left(\tau, \tilde{P}_{\tau}\right)\right)_{p \in[0, \tau]}=\left(V_{p}^{\tau}, \pi_{p}^{\tau}\right)_{p \in[0, \tau]}$, which replicates $\tilde{P}_{\tau}$, i.e:

$$
\left\{\begin{array}{l}
-d V_{p}^{\tau}=b\left(p, V_{p}^{\tau}, \pi_{p}^{\tau}\right) d t-\pi_{p}^{\tau} d W_{s}, 0 \leq p \leq \tau \\
V_{\tau}^{\tau}=\tilde{P}_{\tau}
\end{array}\right.
$$

Therefore, the price is given by the Snell Envelope:

$$
X_{0}=e s s \sup _{\tau} V_{0}\left(\tau, \tilde{P}_{\tau}\right)
$$

Theorem. The value $X_{0}$ is equal to $Y_{0}$, where $(Y, \pi, K)$ is the solution of the following reflected $B S D E$ :

$$
\left\{\begin{array}{l}
-d Y_{t}=b\left(t, Y_{t}, \pi_{t}\right) d t+d K_{t}-\pi_{t} d W_{t}, t \in[0, T] \\
Y_{T}=\xi \\
T_{t} \geq P_{t}, \forall t \in[0, T] \\
\int_{0}^{T}\left(Y_{t}-P_{t}\right) d K_{t}=0
\end{array}\right.
$$

Furthermore, the stopping time:

$$
v:=\inf \left\{s \geq 0 \mid Y_{s}=P_{s}\right\} \wedge T,
$$

is the optimal exercise time, namely $X_{0}=V_{0}\left(v, \tilde{P}_{v}\right)$.

Proof. The existence of a solution $(Y, \pi, K) \in L_{a d}^{2}(\Omega \times[0, T]) \times L_{a d}^{2}(\Omega \times$ $\left.[0, T] ; \mathbb{R}^{d}\right) \times S^{2}([0, T])$ of the RBSDE is guaranteed by the existence and uniqueness result. Moreover, if $\tau$ is a stopping time such that $\tau \leq T$, then we have:

$$
\left\{\begin{array}{l}
-d V_{p}^{\tau}=b\left(p, V_{p}^{\tau}, \pi_{p}^{\tau}\right) d t-\pi_{p}^{\tau} d W_{p}, 0 \leq p \leq \tau \\
V_{\tau}^{\tau}=\tilde{P}_{\tau}
\end{array}\right.
$$

Since $-d Y_{s}=b\left(s, Y_{s}, \pi_{s}\right) d s+d K_{s}-\pi_{s} d W_{s}, s \in[0, \tau]$, and $Y_{\tau} \geq \tilde{P}_{\tau}$, then, by the comparison principle, we have:

$$
Y_{s} \geq V_{s}^{\tau}, \forall s \in[0, \tau], P-a . s .
$$

We can view equation (5) as a reflected BSDE, with barrier $V_{t}^{\tau} \wedge \tilde{P}_{t} ; t \in[0, \tau]$, hence:

$$
Y_{0} \geq e s s \sup _{\tau} V_{0}\left(\tau, \tilde{P}_{\tau}\right)
$$

On the other hand we have:

$$
-d Y_{t}=b\left(t, Y_{t}, \pi_{t}\right) d t-\pi_{t} d W_{t} ; \forall t \in[0, v],
$$

with $Y_{v}=\tilde{P}_{v}$, so that $Y_{0}=V_{0}\left(v, \tilde{P}_{v}\right) \leq e s s \sup _{\tau} V_{0}\left(\tau, \tilde{P}_{\tau}\right)$. 


\subsection{RBSDEs-Quantization Approach to American Option Pricing}

In what follows, mainly exploiting the results given in [2], see also [7] and references therein, we describe a probabilistic method for pricing American style options on multidimensional baskets of traded assets and we see the connection between this method and RBSDEs. First of all the asset dynamics follow a $d$-dimensiona, $d \geq 1$, diffusion model in the time interval $[0, T]$, where $T$ is the maturity time. Taking into account a continuous time Markov process $\left(S_{t}\right)_{t \in[0, T]}$ for the underlying, the pricing procedure consists in solving the continuous optimal stopping time problem related to an obstacle process, typically of the form $h_{t}=h\left(t, S_{t}\right)$.

In order to apply a quantization method based on grids, we first discretize the asset price process at times $t_{k}:=k T / n$, where $k=0, \ldots, n$, as explained in [2], then we simulate large samples of $\left(S_{t_{k}}\right)_{0 \leq k \leq n}$ to produce at each time $t_{k}$ a grid $\Gamma_{k}$ of size $N_{k}$, which is optimally fitted to $S_{t_{k}}$, among all grids with size $N_{k}$, namely the closest neighbor rule projection $q_{\Gamma_{k}}\left(S_{t_{k}}\right)$ of $S_{t_{k}}$ onto the grid $\Gamma_{k}$ is the best least square approximation of $S_{t_{k}}$ among all random vectors $Z$ s.t. $|Z(\Omega)| \leq N_{k}$, so that

$$
\left\|S_{t_{k}}-q_{\Gamma_{k}}\left(S_{t_{k}}\right)\right\|_{2}=\min \left\{\left\|S_{t_{k}}-Z\right\|_{2}, Z: \Omega \rightarrow \mathbb{R}^{d},|Z(\Omega)| \leq N_{k}\right\} .
$$

Following such an approach, like in [2], we will work with the best possible grid of size $N_{k}$, at each time step $t_{k}$, to approximate the $d$-dimensional random vector $S_{t_{k}}$. Both the function $q_{\Gamma_{k}}$ and the set $q_{\Gamma_{k}}(\Omega)$ are called optimal quantizer of $S_{t_{k}}$ with resulting error bound represented by $\left\|S_{t_{k}}-q_{\Gamma_{k}}\left(S_{t_{k}}\right)\right\|_{2}$, the latter being called the lowest quantization error, in the quadratic mean sense.

Hence the model is constructed in the following way: we consider a market on which are traded $d \geq 1$ risky assets $S^{1}, \ldots, S^{d}$, and a deterministic riskless asset $S_{t}^{0}:=e^{r t}, r \in \mathbb{R}$, in a finite time interval $[0, T], T>0$. The price process $S:=\left(S^{1}, \ldots, S^{d}\right)$ for the risky assets is taken to have the following structure:

$$
d S_{t}^{i}=S_{t}^{i}\left(r d t+\sum_{1 \leq j \leq q} \sigma_{i j}\left(e^{-r t} S_{t}\right) d W_{t}^{j}\right), S_{0}^{i}:=s_{0}^{i}>0,1 \leq i \leq d
$$

where $W:=\left(W^{1}, \ldots, W^{q}\right)$ is a $q$-dimensional Brownian Motion defined on a probability space $(\Omega, F, \mathbb{P})$ and $\sigma: \mathbb{R}^{d} \rightarrow M(d \times q):=\mathbb{R}^{d \otimes q}$ is bounded and Lipschitz continuous. The associated filtration is denoted by $F:=\left(F_{t}^{s}\right)_{t \in[0, T]}$, and it is the one generated by $S$, which coincides with that of $W$ when $\sigma \sigma^{T}(\xi)>$ 0 for every $\xi \in \mathbb{R}^{d}$. Moreover we introduce:

$$
c(\xi):=\operatorname{Diag}(\xi) \sigma(\xi), \xi:=\left(\xi^{1}, \ldots, \xi^{d}\right) \in \mathbb{R}^{d},
$$


where $\operatorname{Diag}(\xi)$ denotes the diagonal matrix with diagonal entry $\xi^{i}$ at row $i$. Assuming that the functions $c(\xi)$, and the drift $b(\xi):=r \xi$, are Lipschitz continuous, we have the existence and uniqueness of the solution for $(6)$ on $(\Omega, F, \mathbb{P})$. The discounted price process $\tilde{S}_{t}:=e^{-r t} S_{t}$ is a positive $\mathbb{P}$-martingale satisfying:

$$
d \tilde{S}_{t}=c\left(\tilde{S}_{t}\right) d W_{t}, \tilde{S}_{0}:=s_{0} .
$$

In what follows we assum that $\mathbb{P}$ represents the risk neutral probability of the model we are considering. When $q \neq d$, the completeness of the market fails, nevertheless, this does not influence the implementation of the quantization method to compute the price of the derivatives. When $q=d$, and $\sigma(x) \equiv \sigma \in$ $M(d \times d)$, eq. (6) represents the usual $d$-dimensional Black \& Scholes model, namely the risky assets follow a geometric Brownian motions whose dynamics are given by:

$$
S_{t}^{i}=s_{0}^{i} \exp \left(\left(r-\frac{1}{2}\left|\sigma_{i}\right|^{2}\right) t+\sum_{1 \leq j \leq d} \sigma_{i j} W_{t}^{j}\right), 1 \leq i \leq d .
$$

Let us now take into consideration an American option related to a payoff process $\left(h_{t}\right)_{t \in[0, T]}$, which is a $F$-adapted nonnegative process. We will always consider the sub-class of payoffs $h_{t}$ that only depend on $\left(t, S_{t}\right)$, i.e. they satisfy:

$$
h_{t}:=h\left(t, S_{t}\right), t \in[0, T], h:[0, T] \rightarrow \mathbb{R}_{+} \text {being Lipschitz continuous . }
$$

Under the assumptions (6) and (8), one has:

$$
\mathbb{E}\left(\sup _{t \in[0, T]}\left|h_{t}\right|^{p}\right)<+\infty, \forall p \geq 1 .
$$

In a complete market, the fair price $\nu_{t}$ at time $t$ is:

$$
\left.\nu_{t}:=e^{r t} e s s \sup \left\{\mathbb{E}\left(e^{-r t} h_{\tau} \mid F\right), \tau \in\right\rceil_{t}\right\},
$$

where $\rceil_{t}:=\{\tau: \Omega \rightarrow[t, T], F$-stopping time $\}$. The latter simply means that the discounted price of the option represented by $\tilde{\nu}_{t}:=e^{-r t} \nu_{t}$, is nothing but the Snell envelope of the discounted American payoff:

$$
\tilde{h}_{t}:=\tilde{h}\left(t, \tilde{S}_{t}\right), \text { with } \tilde{h}(t, x):=e^{-r t} h\left(t, e^{r t} x\right) .
$$

Using the Markov property of the diffusion process $\left(S_{t}\right)_{t \in[0, T]}$, it is possible to show that, see [2], $\nu_{t}:=v\left(t, S_{t}\right)$, where $v$ solves the variational inequality:

$$
\max \left(\frac{\partial v}{\partial t}+L_{r, \sigma} v, v-h\right)=0, v(T, \cdot)=h(T, \cdot),
$$


$L_{r, \sigma}$ being the infinitesimal generator associated to eq. (6). Therefore the approximation problem for $\nu_{t}$ appears as a special case of the Snell envelope of a $d$-dimensional diffusion with Lipschitz coefficients.

Following [2], we assum without loss of generality, that the interest rate $r$ in eq. (6) is 0, hence are in the so called discounted world setting, with $\tilde{S}$ given by (7), and $\tilde{h}$ given by (10), instead of $S_{t}$ and $h$, respectively.

The peculiarity of the martingale diffusion dynamics proposed for the risky assets by eq. (7), and with $r=0$, can be shown to have little influence on the results, hence we can consider a general drifted Brownian diffusion of the following type

$$
S_{t}=S_{0}+\int_{0}^{t} b\left(S_{s}\right) d s+\int_{0}^{t} c\left(S_{s}\right) d W s,
$$

where $b: \mathbb{R}^{d} \rightarrow \mathbb{R}^{d}$, and $c: \mathbb{R}^{d} \rightarrow M(d \times q)$ are both Lipschitz continuous vector fields, and $\left(W_{t}\right)_{t \in[0, T]}$ is $q$-dimensional Brownian motion. When $\sigma$ is not constant, for example, in the model described by eq. (6), the exact simulation of a diffusion at time $t$ is not feasible, and it is customary to implement a Markovian discretization scheme, as, e.g., the Euler scheme. The latter consists in setting $t_{k}=k T / n$, so that

$$
\bar{S}_{t_{k+1}}=\bar{S}_{t_{k}}+b\left(\bar{S}_{t_{k}}\right) \frac{T}{n}+c\left(\bar{S}_{t_{k}}\right) \cdot\left(W_{t_{k}}-W_{t_{k-1}}\right), \bar{S}_{0}=s_{0} 1 ;
$$

Sometimes, the diffusion can be simulated in a rather straightforward manner, essentially because it can be expressed in a closed form, e.g. $S_{t}:=\phi\left(t, W_{t}\right)$. This is the case of the regular multi-dimensional Black \& Scholes model, taking $\sigma(x):=\sigma$ in (6), which allows to consider directly the Snell envelope of the homogeneous Markov chain $\left(S_{t_{k}}\right)_{0 \leq k \leq n}$, for our quantization aims.

Latter procedure corresponds, from the derivatives point of view, to approximate the original continuous time American option by a Bermuda option, characterized by a finite set of exercise times, either on $\bar{S}$, or on $S$ itself. Moreover the Snell envelope can be quantized considering $\left(S_{t_{k}}\right)$ or $\left(\bar{S}_{t_{k}}\right)$, or of any family of homogeneous discrete time $F_{t_{k}}$-Markov chains $\left(X_{k}^{(n)}\right)_{0 \leq k \leq n}$ whose transition, denoted $P^{(n)}(x, d y)$, preserves the Lipschitz continuity property, at least in the following sens: for every Lipschitz continuous function $f: \mathbb{R}^{d} \rightarrow \mathbb{R}$ we require that

$$
\left[P^{(n)} f\right]_{\text {Lip }} \leq\left(1+C_{b, \sigma, T} T / n\right)[f]_{\text {Lip }}, \quad[f]_{\text {Lip }}:=\sup _{x \neq y} \frac{|f(x)-f(y)|}{|x-y|} .
$$

To simplify notations, we drop the dependency in $n$, keeping just $\left(X_{k}\right)_{0 \leq k \leq n}$. 
The $\left(F_{t_{k}}\right)$-Snell envelope of $h\left(t_{k}, X_{k}\right)$, denoted by $\left(V_{k}\right)_{0 \leq k \leq n}$, is defined by:

$$
V_{k}:=e s s \sup \left\{\mathbb{E}\left(h\left(\theta, X_{\theta}\right) \mid F_{t_{k}}\right), \theta \in \Theta_{k}\right\},
$$

where $\Theta_{k}$ denotes the set of $\left\{t_{k}, \ldots, t_{n}\right\}$-valued $\left(F_{t_{l}}\right)$-stopping times; it satisfies the backward dynamic programming formula:

$$
\left\{\begin{array}{l}
V_{n}:=h\left(t_{n}, X_{n}\right) \\
V_{k}:=\max \left(h\left(t_{k}, X_{k}\right), \mathbb{E}\left(V_{k+1} \mid F_{t_{k}}\right)\right) \quad 0 \leq k \leq n-1
\end{array} .\right.
$$

Exploiting the Markov property, it is possible to derive a dynamic programming formula in distribution: $V_{k}=v_{k}\left(X_{k}\right), k \in\{0, \ldots, n\}$, where the functions $v_{k}$ are recursively defined as follow

$$
\left\{\begin{array}{l}
v_{n}:=h\left(t_{n}, \cdot\right) \\
v_{k}:=\max \left(h\left(t_{k}, \cdot\right), P^{(n)}\left(v_{k+1}\right)\right) \quad 0 \leq k \leq n-1
\end{array},\right.
$$

nevertheless, from numerical point of view, such type of formula are intractable, since they require to compute at each time step a conditional expectation.

Hence we are in position to describe the quantization method core. In particular, we first discretize the random variables $X_{k}$ by some $\sigma\left(X_{k}\right)$-random variables $\hat{X}_{k}$ taking finite values in $\mathbb{R}^{d}$, so that it is natural to define $\hat{X}_{k}$ as the quantized counterpart of $X_{k}$. Then, being $N=N_{0}+N_{1}+\ldots+N_{n}$ the total number of elementary quantizers used to quantize the whole Markov chain $\left(X_{k}\right)_{0 \leq k \leq n}$. Moreover we assume that, $\forall k, \in\{0,1, \ldots, n\}$, we have a sequence of quantizations $\hat{X}_{k}=q_{k}\left(X_{k}\right), k=0, \ldots, n$ of the Markov chain $\left(X_{k}\right)_{k}$, and we also denote by $\left\{x_{1}^{k}, \ldots, n_{N_{k}}^{k}\right\}=q_{k}\left(\mathbb{R}^{d}\right)$, the grid of $N_{k}$ points used to quantize $X_{k}$, and by $x^{k}=\left(x_{1}^{k}, \ldots, x_{N_{k}}^{k}\right)$ the induced $N_{k^{-}}$tuple.

Then we can state the quantized dynamic programming formula below, in tight analogy with eq. (11). In particular, one simply replaces $X_{k}$ by its quantized random vector $\hat{X}_{k}$ :

$$
\left\{\begin{array}{l}
\hat{V}_{n}:=h\left(t_{n}, \hat{X}_{n}\right) \\
\hat{V}_{k}:=\max \left(h\left(t_{k}, \hat{X}_{k}\right), \mathbb{E}\left(\hat{V}_{k+1} \mid \hat{X}_{k}\right)\right) \quad 0 \leq k \leq n-1
\end{array} .\right.
$$

In what follows, for the sake of simplicity, we will use the following notation $\hat{\mathbb{E}}_{k}(\cdot):=\mathbb{E}\left(\cdot \mid \hat{X}_{k}\right)$. Since $\left(\hat{X}_{k}\right)_{k \in \mathbb{N}}$ is not Markovian, we consider the conditional expectation with respect to $\hat{X}_{k}$. On the other hand, even if the $N_{k}$-tuple $x^{k}$ has been set up a priori for every $X_{k}$, the numerical processing of this algorithm is not possible. Indeed, one needs to know the joint distributions of $\left(\hat{X}_{k}, \hat{X}_{k+1}\right), k=0, \ldots, n-1$, as shown by the the following proposition, see [2]: 
Proposition (Quantization tree algorithm) For every $k \in\{0, \ldots, n\}$, let $x^{k}:=\left(x_{1}^{k}, \ldots, x_{N_{k}}^{k}\right), q_{k}: \mathbb{R}^{d} \rightarrow\left\{x_{1}^{k}, \ldots, x_{N_{k}}^{k}\right\}$ and $\hat{X}_{k}=q_{k}\left(X_{k}\right)$ be a quantization of $X_{k}$. Set, for every $k \in\{0, \ldots, n\}$ and every $i \in\left\{1, \ldots, N_{k}\right\}$ :

$$
p_{i}^{k}:=\mathbb{P}\left(\hat{X}_{k}=x_{i}^{k}\right)=\mathbb{P}\left(X_{k} \in C_{i}\left(x^{k}\right)\right)
$$

and, for every $k \in\{0, \ldots, n-1\}, i \in\left\{1, \ldots, N_{k}\right\}, j \in\left\{1, \ldots, N_{k+1}\right\}$ :

$$
\begin{aligned}
\pi_{i, j}^{k}:= & \mathbb{P}\left(\hat{X}_{k+1}=x_{j}^{k+1} \mid \hat{X}_{k}=x_{i}^{k}\right)=\mathbb{P}\left(X_{k+1} \in C_{j}\left(x^{k+1}\right) \mid X_{k} \in C_{i}\left(x^{k}\right)\right)= \\
= & \frac{p_{i j}^{k}}{p_{i}^{k}} \text { with } p_{i j}^{k}:=\mathbb{P}\left(X_{k+1} \in C_{j}\left(x^{k+1}\right), X_{k} \in C_{i}\left(x^{k}\right)\right),
\end{aligned}
$$

then, by backward induction, one defines the function $\hat{v}_{k}$ by:

$$
\begin{gathered}
\hat{v}_{n}\left(x_{i}^{n}\right):=h_{n}\left(x_{i}^{k}\right), i \in\left\{1, \ldots, N_{n}\right\}, \\
\hat{v}_{k}\left(x_{i}^{k}\right):=\max \left(h\left(t_{k}, x_{i}^{k}\right), \sum_{j=1}^{N_{k+1}} \pi_{i j}^{k} \hat{v}_{k+1}\left(x_{j}^{k+1}\right)\right), 1 \leq i \leq N_{k}, 0 \leq k \leq n-1 .
\end{gathered}
$$

Therefore $\hat{V}_{k}=\hat{v}_{k}\left(\hat{X}_{k}\right)$ satisfies the above dynamic programming (12) of the pseudo-Snell envelope.

Remark. If $X_{k}=S_{t_{k}}$ or $\bar{S}_{t_{k}}$, then $X_{0}=\hat{X}_{0}=s_{0}$ and $\hat{v}_{0}\left(\hat{X}_{0}\right)=\hat{v}_{0}\left(s_{0}\right)$ is deterministic. In more general settings one approximates $\mathbb{E}\left[v_{0}\left(X_{0}\right)\right]$ by:

$$
\mathbb{E}\left[\hat{v}_{0}\left(\hat{X}_{0}\right)=\sum_{i=1}^{N_{0}} p_{i}^{0} \hat{v}\left(x_{i}^{0}\right) .\right.
$$

The RBSDEs theory comes into play when we want to evaluate the global residual risk on time interval $\left[0, T^{\prime}\right], T^{\prime}<T$, which is induced by the use of the time discretization of the diffusion with step $T / n$, namely:

$$
\mathbb{E}\left[\int_{0}^{T-\delta}\left|c^{*}\left(S_{s}\right)\left(Z_{s}-\zeta_{s}\right)\right|^{2} d s\right]
$$

where $Z_{t}:=\left[c\left(S_{t}\right)^{*}\right]^{-1} H_{t}, H_{t}$ being an adapted process, while $\zeta_{t}:=\operatorname{proj}_{n}(Z)$ is the projection on the set $P_{n}$ of elementary predictable strategies. Our basic assumption, let us denote it by $(\Sigma)$, reads as follows:

(i) $c \in C_{b}^{\infty}\left((0,+\infty)^{d}\right)$,

(ii) $\sigma \sigma^{*}(x) \geq \epsilon_{0} I_{d}$. 
Assumption (i) is fulfilled when $\sigma \in C_{b}\left((0,+\infty)^{d}\right) \cap C_{b}^{\infty}\left((0,+\infty)^{d}\right)$ and:

$$
\partial^{k} \sigma_{i} \cdot(x)=O\left(1 /\left|x^{i}\right|\right) \text { as }|x| \rightarrow+\infty k \geq 1, i=1, \ldots, d .
$$

The difficulties arise when we want evaluate the term in (16) directly. So, like in [2], we reduce the problem to a simpler one, according to the following two steps:

STEP 1. Lemma. Set $H_{s}:=c^{*}\left(S_{s}\right) \cdot Z_{s}$ and $\eta_{s}:=\frac{n}{T} \mathbb{E}_{t_{k}}\left[\int_{t_{k}}^{t_{k+1}} H_{u} d u\right], s \in$ $\left[t_{k}, t_{k+1}\right]$. Then, under the assumptions of the previous theorem, we have

$$
\mathbb{E}\left[\int_{0}^{T}\left|c^{*}\left(S_{s}\right)\left(Z_{s}-\zeta_{s}\right)\right|^{2} d s\right] \leq \frac{C}{n}+2 \mathbb{E}\left[\int_{0}^{T}\left|H_{s}-\eta_{s}\right|^{2} d s\right],
$$

as stated in [2, Subsec. 4.3, Lemma 1].

STEP 2. The second type of difficulty we have to take into account is due to the following two facts

- the obstacle $h\left(t, S_{t}\right)$ is not sufficiently smooth, then we do not have a nice control on the increasing process $K_{t}$;

- the process $S_{t}$ is not uniformly elliptic, since $c(0)=0$, then we cannot count on nice evaluations for the (probability) density of $S_{t}$.

In order to overcome previous issues, we will replace $S$ by an elliptic diffusion denoted $\underline{\mathrm{S}}$ and the obstacle $h$ by a smoother one, denoted by $\underline{\mathrm{h}}$. Let $\epsilon \in(0,1]$ and $\lambda>0$, then we consider:

- a function $\underline{\mathrm{h}} \in C^{1,2}\left(\mathbb{R}_{+} \times \mathbb{R}^{d}, \mathbb{R}\right)$, with a regularization by convolution of order $\epsilon$ of the original obstacle $h$, then, since $h$ is Lipschitz continuous, we have:

$$
\|h-\underline{\mathrm{h}}\|_{\infty} \leq C_{h} \epsilon \text { and }\left\|\left(\partial_{t}+L_{c}\right) \underline{\mathrm{h}}\right\|_{\infty} \leq C_{h} \epsilon^{-1},
$$

where $L_{c}$ is the infinitesimal generator of the diffusion $\mathrm{S}$.

— a function $\phi_{\lambda} \in C_{b}^{\infty}(\mathbb{R}, \mathbb{R})$ satisfying:

$$
\phi_{\lambda}(\xi):=\xi \text { if } \xi \geq e^{-\lambda}, \phi_{\lambda}(\xi):=\frac{e^{-\lambda}}{2} \text { if } \xi \leq \frac{1}{2} e^{-\lambda},
$$

and

$$
\left\|\phi_{\lambda}^{(m)}\right\|_{\infty} \leq C_{m} e^{C_{m} \lambda}, \forall m \geq 1
$$


where $C_{m}$ is a real constant which does not depend on $\lambda$. Then the approximating diffusion coefficient $c_{\lambda}$, which is defined for every $x=\left(x^{1}, \ldots, x^{d}\right) \in \mathbb{R}^{d}$ by:

$$
c_{\lambda}(x):=c\left(\phi_{\lambda}\left(x^{1}\right), \ldots, \phi_{\lambda}\left(x^{d}\right)\right)
$$

satisfies:

$$
c_{\lambda} \in C_{b}^{\infty}\left(\mathbb{R}^{d}\right) \text { and } c_{\lambda} c_{\lambda}^{*}(x) \geq \frac{\epsilon_{0}}{4} e^{-2 \lambda} I_{d}
$$

Taking into consideration the solution $\underline{S}^{x}$ of the SDE:

$$
d \underline{S}_{t}^{x}=r \underline{S}_{t}^{x} d t+c_{\lambda}\left(\underline{\mathrm{S}}_{t}^{x}\right) d W_{t}, \underline{\mathrm{S}}_{0}^{x}=x,
$$

and being $\underline{\mathrm{P}}_{t}(x, d y)$ its Markov semi-group, defined by $\underline{\mathrm{P}}_{t} f(x)=\mathbb{E}\left[f\left(\underline{\mathrm{S}}_{t}^{x}\right)\right]$, we will denote by $\underline{S}_{t}$ the associated solution $\underline{S}^{s_{0}}$, which starts at $s_{0} \in(0,+\infty)^{d}$, at time $t=0$. Then, the related Snell envelope reads as follow:

$$
\underline{\mathrm{Y}}_{t}=e s s \sup _{\tau \in \mathrm{T}_{t}} \mathbb{E}_{t}\left[\underline{\mathrm{h}}\left(\tau, \underline{\mathrm{S}}_{\tau}\right)\right]
$$

and it satisfies the following RBSDE:

$$
\underline{\mathrm{Y}}_{t}=\underline{\mathrm{h}}\left(T, \underline{\mathrm{S}}_{T}\right)+\underline{\mathrm{K}}_{T}-\underline{\mathrm{K}}_{t}-\int_{t}^{T} \underline{\mathrm{H}}_{s} \cdot d W_{s}
$$

for some non decreasing process $\underline{\mathrm{K}}$ and some progressively, measurable $d \mathbb{P} \otimes d t$ square integrable process $\underline{\mathrm{H}}$. Finally we consider the approximation:

$$
\underline{\mathrm{w}}_{s}=\frac{n}{T} \mathbb{E}_{t_{k}}\left[\int_{t_{k}}^{t_{k+1}} \underline{\mathrm{H}}_{s} d s\right], t_{k} \leq s<t_{k+1},
$$

and we have, see [2, Subsect. 4.5, Lemma2], that the following holds by stability properties of RBSDEs:

Lemma. Assume that $(\Sigma)$ holds. Then:

$$
\mathbb{E}\left[\int_{0}^{T}\left|H_{s}-\eta_{s}\right|^{2} d s\right] \leq C\left(\epsilon^{2}+\left(1+\left|s_{0}\right|\right)^{2} e^{\frac{-C \lambda^{2}}{T}}\right)+\mathbb{E}\left[\int_{0}^{T}\left|\underline{H}_{s}-\underline{w}_{s}\right|^{2} d s\right] .
$$




\subsection{Pricing American Option by Monte Carlo Method}

Before enter into details, let us recall some useful notions and results about the Monte Carlo (MC) method. First of all, let us underline that the main goal of the $\mathrm{MC}$ approach is to compute quantities of the following type

$$
\tau=\mathbb{E}[\phi(X)]=\int_{A} \phi(x) f(x) d x,
$$

where:

- $\mathrm{X}$ is a random variable taking values in $A \subseteq \mathbb{R}^{d}, d \in \mathbb{N}$;

- $f: A \rightarrow \mathbb{R}_{+}$is the probability density of $X$;

- $\phi: A \rightarrow \mathbb{R}_{+}$is a function such that the above expectation is finite;

Latter framework actually covers a large set of fundamental problems in statistics, numerical analysis, mechanics, engineering, etc., see, e.g., $[15,16]$, and references therein. As an example, if we want to compute an integral of the following type

$$
\alpha=\int_{0}^{1} f(x) d x,
$$

we can instead consider the expectation $\mathbb{E}[f(U)]$, with $U$ uniformly distributed between 0 and 1 . Then we, randomly, generate points $U_{1}, U_{2}, \ldots, U_{n}$, independently and uniformly over the interval $[0,1]$, and, by evaluating $f$ at each $U_{i}$ and by taking the associated mean, we get the following $\mathrm{MC}$ estimate

$$
\hat{\alpha}=\frac{1}{n} \sum_{i=1}^{n} f\left(U_{i}\right),
$$

which, by the Law of Large Numbers (LLN), will converge to the true value when $n \rightarrow+\infty$.

In particular the LLN tells us that the expectation and the aritmetic mean, in the above reported sense, are equal in the limit. Moreover, by the Central Limit Theorem (CLT) we can deduce that the distribution of the mean is normal, regardless of the initial distribution of the random variable $X$.

If we want to use a $\mathrm{MC}$ approach to approximate the dynamic of a given stochastic process, see, e.g. [34], we easily see that such MC methods strongly depends on the possibility to perform good simulation of the underlying process. In particular, when we consider a 1-dimensional American option with constant 
interest rate, the underlying process is the stock price $S$ which, taking the risk-free interest rate $r$, and both the parameters $\delta$ and $\sigma$ to be constant, is characterized by the following SDE

$$
d S_{t}=(r-\delta) S_{t} d t+\sigma S_{t} d W_{t}
$$

Let $S_{0}$ be the value of the stock at initial time $t=0$, then we have

$$
S_{t}=S_{0} \exp \left(\left(r-\delta-\frac{\sigma^{2}}{2}\right) t+\sigma W_{t}\right) .
$$

Since the increments of the standard Brownian motion $W$, which drives the dynamic of $S$, are independent and normally distributed, we can simulate paths of the process $S$ using a recursive procedure of the following type

$$
S_{t}=S_{t-1} \exp \left(\left(r-\delta-\frac{\sigma^{2}}{2}\right) d t+\sigma \sqrt{d t} Z\right),
$$

where we have assumed that the time steps are equidistant, and of length $d t$, while $Z \sim N(0,1)$. Latter recursive procedure allows to generate sample paths $S^{i}=\left\{S_{1}^{i}, S_{2}^{i}, \ldots, S_{n}^{i}\right\}, i=1, \ldots, N$.

Most MC methods are exploited for pricing American option with respect to the dynamic programming setting, see [5] for its seminal definition by Bellman.

In particular, let $\Pi_{i}(s)$ denotes the value of the option at time $t_{i}$, given $S_{i}=s$, and assuming that the option has not been exercised. Finally, assume that $\Phi(s)$ is a payoff function of an American-style contract and our aim is to compute the value of $\Pi_{0}\left(S_{0}\right)$. The value of $\Pi_{0}\left(S_{0}\right)$ can be obtained recursively as follows

$$
\begin{gathered}
\Pi_{T}(s)=\Phi_{T}(s) \\
\Pi_{i}(s)=\max \left(\Phi_{i}(s), C_{i}(s)\right), i=0, \ldots, T-1,
\end{gathered}
$$

where $C_{i}(s)$ is the continuation value defined by

$$
C_{i}(s)=\mathbb{E}\left[\Phi\left(S_{i+1}\right) \mid S_{i}=s\right] .
$$

Again citing [34], we have that The dynamic programming formulation thus boils down to at each time step $t=1, \ldots, T-1$, compare the payoff with the expected value of the option if not exercised at this time. Nevertheless, we do not know the continuation value, which, indeed, has to be estimated. In order to do the latter, several approaches are possible, as, e.g., the Random Tree Method, the Stochastic Mesh Method, the Duality Method, the Forward Backwards Method (FBM),etc., see[34, Sec.4]. 
In what follows we shall focus our attention on the FBM. Therefore the continuation value is estimated by a single value, $\hat{\Pi}\left(t+1, B_{t+1}^{i}\right)$. The idea behind the FBM is to construct a grid $B$, by mean of backwards simulation and conditional on the initial value $S_{0}$ of the asset at time $t=0$. Therefore, the value $\hat{\Pi}\left(t+1, B_{t+1}^{i}\right)$ will be an unbiased estimator of the continuation value and the computational cost is quadratic in the number of points in the grid, while it is linear with respect to the number of exercise dates, see [34]. If we assume that $B=\left\{B_{1}^{i}, \ldots, B_{Y}^{i}\right\}, i=1, \ldots, n$, then the related construction proceeds as follow. First we apply the dynamic programming procedure with:

$$
\hat{\Pi}\left(t, B_{t}^{i}\right)=\left\{\begin{array}{ll}
\Phi\left(t, B_{t}^{i}\right) & t=T \\
\max \left(\Phi\left(t, B_{t}^{i}\right), \hat{\Pi}\left(t+1, B_{t+1}^{i}\right)\right) & t<T
\end{array},\right.
$$

then we define the final estimator

$$
\hat{\Pi}\left(0, S_{0}\right):=\max \left(\Phi\left(0, S_{0}\right), \frac{1}{n} \sum_{i=1}^{n} \hat{\Pi}\left(1, B_{1}^{i}\right)\right),
$$

which is high biased by Jensen's inequality, see [34]. To construct the grid $S$ we first simulate $n$ trajectories for $S$, obtaining $S=\left\{S_{1}^{i}, \ldots, S_{T}^{i}\right\}, i=1, \ldots, n$, then we can consider $S$ as our forward grid and define $B=\left\{B_{1}^{i}, \ldots, B_{T}^{i}\right\}, i=1, \ldots, n$, as the backward one. Therefore, see [34], we have

$$
B_{t}^{i}= \begin{cases}S_{T}^{i} & t=T \\ S_{t}^{I_{t}^{i}} & t<T\end{cases}
$$

where $I_{t}^{i}$ is drawn from $\{1,2, \ldots, n\}$ with probability:

$$
I_{t}^{i} \sim\left(\frac{p\left(S_{t+1}=B_{t+1}^{i} \mid S_{t}=S_{t}^{\ell}\right)}{\sum_{j=1}^{n} p\left(S_{t+1}=B_{t+1}^{i} \mid S_{t}=S_{t}^{j}\right)}\right)_{\ell},
$$

$p$ being the probability density of the underlying $S$, in fact we want to simulate $B_{t}^{i}$ conditional on $S_{0}=s$ and $S_{t+1}^{i}=B_{t+1}^{i}$, namely with probability:

$$
\begin{gathered}
p\left(S_{t} \mid S_{t+1}, S_{0}\right)=\frac{p\left(S_{t}, S_{t+1}, S_{0}\right)}{p\left(S_{t+1}, S_{0}\right)}=\frac{p\left(S_{t}, S_{t+1}, S_{0}\right)}{\int p\left(S_{t}, S_{t+1}, S_{0}\right) d s_{t}}= \\
=\frac{p\left(S_{t+1} \mid S_{t}, S_{0}\right) p\left(S_{t} \mid S_{0}\right) p\left(S_{0}\right)}{\int p\left(S_{t+1} \mid S_{t}, S_{0}\right) p\left(S_{t} \mid S_{0}\right) p\left(S_{0}\right) d s_{t}}
\end{gathered}
$$


and taking into consideration the empirical measure in the denominator of (17), equation (17) simplifies to:

$$
=\frac{p\left(S_{t+1} \mid S_{t}\right)}{\sum_{\ell} p\left(S_{t+1} \mid S_{t}^{\ell}\right)}
$$

because $S$ is (now) a discrete Markov chain, threfore we have the same as in (17).

\section{Conclusion}

In this paper we have underlined how the theory behind the study of RBSDEs can be well adapted to solve the non-linear problem of pricing American option. In particular we have reported the existing link between American option and RBSDEs, namely the price of an American option, according to the notation introduced in Subsection 4.1, is given by the Snell Envelope:

$$
X_{0}=e s s \sup _{\tau} V_{0}\left(\tau, \tilde{P}_{\tau}\right)
$$

and $X_{0}$ equals $Y_{0}$, the latter being the solution of the RBSDE:

$$
\left\{\begin{array}{ll}
-d Y_{t}=b\left(t, Y_{t}, \pi_{t}\right) d t+d K_{t}-\pi_{t} d W_{t} & t \in[0, T] \\
Y_{T}=\xi & \forall t \in[0, T] \\
T_{t} \geq P_{t} & \\
\int_{0}^{T}\left(Y_{t}-P_{t}\right) d K_{t}=0 &
\end{array} .\right.
$$

Then, we have shown how the quantization method can be used to numerically study the solutions of a suitable class of RBSDEs, namely those related to the task of finding the price $v$ of an American option. In particular, according to the notation introduced in Subsection 4.2, we have that $\hat{V}_{k}=\hat{v}_{k}\left(\hat{X}_{k}\right)$ satisfies the dynamic programming principle:

$$
\left\{\begin{array}{l}
\hat{V}_{n}:=h\left(t_{n}, \hat{X}_{n}\right) \\
\hat{V}_{k}:=\max \left(h\left(t_{k}, \hat{X}_{k}\right), \mathbb{E}\left(\hat{V}_{k+1} \mid \hat{X}_{k}\right)\right) \quad 0 \leq k \leq n-1
\end{array}\right.
$$

which is quantized by quantization tree algorithm, where we recall that $\hat{V}$ indicates an approximation of $\mathrm{V}$.

Finally the connection between RBSDEs and Monte Carlo method have been retrivied exploiting the work of Wieslander, see [34], again by using the 
dynamic programming approach, therefore, according to the notation introduced in Subsection 4.3, the price of an American option $\Pi$ is Monte Carlo approximated as follows

$$
\left\{\begin{array}{l}
\Pi_{T}(s)=\Phi_{T}(s) \\
\Pi_{i}(s)=\max \left(\Phi_{i}(s), C_{i}(s)\right) \quad i=0, \ldots, T-1
\end{array} .\right.
$$

We would also like to recall that the RBSDEs theory can be fruitfully applied to different problems, not only related to the Mathematical Finance framweorks, as, e.g., concerning stochastic optimal control, stochastic game, insurance issues, stochastic approches to neuronal dynamics, etc.

\section{References}

[1] V. Bally, M.E. Caballero, B. Fernandez and N. El-Karoui, Reflected BSDE's, PDE's and Variational Inequalities, RR-4455, INRIA. 2002. jinria-00072133i.

[2] V. Bally, G.Pags, J.Printems, A quantization tree method for pricing and hedging multi-dimensional American options, 2001 AMS classification, DOI: 10.1111/j.09601627.2005.00213.x.

[3] V. Barbu, F. Cordoni, and L. Di Persio, Optimal control of stochastic FitzHughNagumo equation, International Journal of Control, vol. 89, no. 4, pp. 746756, DOI: 10.1080/00207179.2015.1096023, 2016.

[4] O. Bardou, S. Bouthemy, G. Pags, Optimal quantization for the pricing of swing options, Applied Mathematical Finance, 16(2):183-217, 2009, DOI: 10.1080/13504860802453218, 2009.

[5] R. Bellman, The theory of dynamic programming, Bull. Amer. Math. Soc. 60 (1954), 503-515, http://projecteuclid.org/euclid.bams/1183519147.

[6] J.M. Bismut, Conjugate Convex Functions in Optimal Stochastic Control, J. Math. Anal. Appl., 44, 384-404 (1973), DOI: 10.1016/0022-247X(73)90066-8.

[7] M. Bonollo, L. Di Persio, I. Oliva and A. Semmoloni, A Quantization Approach to the Counterparty Credit Exposure Estimation, https://arxiv.org/abs/1503.01754 (submitted) 2015 .

[8] M. Bonollo, L. Di Persio and G. Pellegrini, Polynomial chaos expansion approach to interest rate models, Journal of Probability and Statistics Vol. 2015, Art. NO. 369053, http://dx.doi.org/10.1155/2015/369053, 2015.

[9] P. Bossaerts, Simulation estimators of optimal early exercise, 1989.

[10] Phelim P. Boyle, Options: A Monte Carlo approach, Journal of Financial Economics 4 (1977), 1977, DOI: 10.1016/0304-405X(77)90005-8.

[11] F. Cordoni and L. Di Persio, Backward stochastic differential equations approach to hedging, option pricing, and insurance problems, International Journal of Stochastic Analysis, vol. 2014, Article ID 152389, http://dx.doi.org/10.1155/2014/152389, 2014. 
[12] F. Cordoni and L. Di Persio, Invariant measure for the Vasicek interest rate model in the Heath-Jarrow-Morton-Musiela framework, Infinite Dimensional Analysis Quantum Probability and Related Topics, vol. 18, no. 3, http://dx.doi.org/10.1142/S0219025715500228, 2015 .

[13] F. Cordoni and L. Di Persio, A BSDE with Delayed Generator Approach to Pricing under Counterparty Risk and Collateralization, International Journal of Stochastic Analysis, Article ID 1059303, 11 pages, (2016). doi:10.1155/2016/1059303

[14] F. Cordoni, L. Di Persio, L. Maticiuc, and A. Zalinescu, A stochastic approach to pathdependent nonlinear Kolmogorov equations via BSDEs with time-delayed generators and applications to finance, http://arxiv.org/abs/1602.05793. (submitted) 2016.

[15] L. Di Persio and M. Frigo, Gibbs sampling approach to regime switching analysis of financial time series, Journal of Computational and Applied Mathematics, vol. 300, doi:10.1016/j.cam.2015.12.010, 2016

[16] L. Di Persio and M. Frigo, Maximum likelihood approach to markov switching models, WSEAS Transactions on Business and Economics, Volume 12, Pages 239-242, 2015

[17] Duffie, D.; Epstein, L., Stochastic differential utility, Econometrica 60 (1992), no. 2, 353394, DOI: $10.2307 / 2951600$.

[18] Duffie, D.; Epstein, L., Asset Pricing with Stochastic Differential Utility, Rev. Financial Stud. (1992), 5, 411436, DOI: 10.1093/rfs/5.3.411.

[19] El Karoui, Peng, Quenez, Backward Stochastic Differential Equation in Finance, Mathematical Finance, Vol. 7, No. 1 (January 1997), 171, DOI: 10.1111/1467-9965.00022.

[20] El Karoui, N.; Kapoudjian, C.; Pardoux, E.; Peng, S.; Quenez M.C., Reflected Solutions of Backward SDE and Related Obstacle Problems for PDEs, Ann. Probab. Vol. 25 (1997), No. 2, 702-737, DOI: 10.1214/aop/1024404416.

[21] E. H. Essaky, Reflected Backward Stochastic Differential Equation with jumps and RCLL obstacle, DOI: 10.1016/j.bulsci.2008.03.005.

[22] A. Gersho, R. Gray, IEEE on Information Theory, special issue on Quantization, 28, 1982.

[23] S. Hamadne, Reflected BSDE's with Discontinuous Barrier and Application, Laboratoire de Statistique et Processus, Universit du Maine, 72085 Le Mans Cedex 9, DOI: 10.1080/1045112021000036545.

[24] David B. Hertz, Risk analysis in capital investment, Harvard Business Review, January/February issue, 1964. DOI: 10.1225/79504.

[25] C. Labart, J. Lelong, A parallel Algorithm for solving BSDEs-Applicatio to the pricing and hedging of American options, December 2, 2013.

[26] H. Luschgy and G. Pags, Functional quantization of Gaussian processes, Journal of Functional Analysis, 196(2):486-531, 2002, DOI: 10.1016/S0022-1236(02)00010-1.

[27] C. Marinelli, L. Di Persio, and G. Ziglio, Approximation and convergence of solutions to semilinear stochastic evolution equations with jumps, Journal of Functional Analysis, vol. 264, no. 12, DOI: 10.1016/j.jfa.2013.02.020, 2013.

[28] G. Pags, H. Pham, J. Printems, Optimal quantization methods and applications to numerical problems in finance, April 2003, DOI: 10.1007/978-0-8176-8180-7_7. 
[29] G. Pags and J. Printems, Functional quantization for numerics with an application to option pricing, Monte Carlo Methods and Appl., 11(11):407-446, 2005, DOI: 10.1515/156939605777438578.

[30] G. Pags, J. Printems, Optimal quadratic quantization for numerics: the Gaussian case, Monte Carlo Methods and Applications, 9:135-166, 2003, DOI: 10.1515/156939603322663321.

[31] Pardoux, E.; Peng, S., Adapted Solutions of Backward stochastic differential equation, Systems and Control Letters 14 (1990), 55-61, DOI: 10.1016/0167-6911(90)90082-6.

[32] J.L Snell, Application of martingale system theorems, Trans. Am. MAth. Soc., 73, 293312, DOI: 10.1090/S0002-9947-1952-0050209-9.

[33] J.A Tilley, Valuing American options in a path simulation model, Transactions of the Society of Actuaries, 45.

[34] P. Wieslander, On Monte Carlo approximation of the Snell envelope with application to the pricing of American options, https://lup.lub.lu.se/studentpapers/search/publication/4618206, 2013. 